Изв. АН Эстонии. Физ. Матем., 1989, 38, № 4, 403-410

удК $62-504.6$

H. $A C T P O B$

\title{
УРАВНЕНИЯ В ПЕРЕМЕННЫХ СОСТОЯНИЯ ДВУХСКОРОСТНОГО МНОГОМЕРНОГО ДИСКРЕТНОГО ОБЪЕКТА УПРАВЛЕНИЯ
}

\author{
(Представил Ю. Яаксоо)
}

\section{1. Введение}

Текущий этап развития методов цифровой обработки сигналов характеризуется переходом от централизованной к распределенной обработке информации на многих управляющих микро-ЭВМ.

Среди многих известных в настоящее время методов децентрализации особое место занимает метод сингулярных возмущений $\left[{ }^{1}\right]$, так как он дает большой простор для упрощения модели системы и для получения иерархически подразделенных фильтров и регуляторов. Систематический подход для полного разделения «быстрых» и «медленных» конструкций регуляторов, заимствованный из теории сингулярно-возмущенных систем, в данной работе развит расширением идеи раздельного расположения собственных значений $\left[{ }^{2}\right]$ для определенного класса дискретных систем, имеющих свойство разделения во времени.

Класс получаемых в результате децентрализации систем управления в данной работе ограничен двухскоростными многомерными дискретными линейными системами, т. е. системами, которые состоят из двух независимых подсистем («медленной» и «быстрой»), имеющих каждая свой, отличный от другого период дискретности (большой и малый соответственно). Известно, что свойства дискретных систем сильно зависят от выбора периода дискретности. Поэтому определение подходящих периодов дискретности двухскоростных дискретных систем является одной из основных задач при получении их уравнений состояния.

\section{2. Уравнения состояния для эквивалентной дискретной системы}

Рассмотрим непрерывную многомерную систему вида

$$
\begin{aligned}
& \dot{\mathbf{x}}(\tau)=A \mathbf{x}(\tau)+B \mathbf{u}(\tau), \\
& \mathbf{y}(\tau)=C \mathbf{x}(\tau),
\end{aligned}
$$

где $\mathbf{x}-n$-вектор состояния, $\mathbf{u}-m$-вектор входов, $\mathbf{y}-p$-вектор выходов системы. Матрицы $A, B, C$ имеют размерность $n \times n, n \times m$ и $p \times n$ соответственно.

Эквивалентная дискретная система (т. е. система, объединяющая непрерывную систему с аналого-цифровым преобразователем (АЦП) типа импульсного элемента и с цифро-аналоговым преобразователем (ЦАП) типа фиксатора нулевого порядка) может быть описана следующими уравнениями $\left[{ }^{3}\right]$

$$
\begin{aligned}
\mathbf{x}(t+1) & =F \mathbf{x}(t)+G \mathbf{u}(t), \\
\mathbf{y}(t) & =H \mathbf{x}(t), \quad \mathbf{t}=0,1,2, \ldots,
\end{aligned}
$$

где $F=\exp (A \Delta)$, 


$$
G=\left[\int_{0}^{\Delta} \exp (A \eta) d \eta\right] B
$$

$$
H=C \text {, }
$$

без ограничения общности полагаем период дискретности $\Delta=1$.

\section{3. Преобразования уравнений состояния эквивалентной дискретной системы}

Применим метод канонических преобразований $\left[{ }^{4}\right]$ к эквивалентной дискретной системе вида (3)-(4) для получения ее уравнений состояния в канонической форме Жордана. Введем неособое линейное преобразование

$$
\mathbf{q}(t)=T \mathbf{x}(t), \quad \operatorname{det} T \neq 0,
$$

с помощью которого исходную систему (3)-(4) можно переписать в таком виде

$$
\begin{aligned}
\mathbf{q}(t+1) & =J_{d} \mathbf{q}(t)+\tilde{G} \mathbf{u}(t), \\
\mathbf{y}(t) & =\tilde{H} \mathbf{q}(t), \quad t=0,1,2, \ldots,
\end{aligned}
$$

где

$$
J_{d}=T F T^{-1}, \quad T G=\widetilde{G}, \quad H T^{-1}=\mathscr{H} .
$$

Матрицу $J_{d}$ в общем виде можно записать так

$$
J_{d}=\operatorname{diag}\left[J_{k_{1}}\left(z_{1}\right), \ldots, J_{k_{p}}\left(z_{p}\right), J_{k_{p+1}}\left(z_{p+1}, v_{p+1}\right), \ldots, J_{k_{m}}\left(z_{m}, v_{m}\right)\right] \text {. }
$$

Здесь согласно $\left[{ }^{5}\right] J_{h_{t}}\left(z_{i}\right)$ - жорданова клетка, соответствующая действительному полюсу системы $z_{i}$ кратности $k_{i}$

$$
J_{k_{t}}\left(z_{i}\right)=\left[\begin{array}{ccccc}
z_{i} & 1 & 0 & \ldots & 0 \\
0 & z_{i} & 1 & \ldots & 0 \\
0 & 0 & z_{i} & \ldots & 0 \\
\ldots & \ldots & \ldots & \ldots & 0 \\
0 & 0 & 0 & \ldots & z_{i}
\end{array}\right]_{k_{\iota} \times k_{t}}
$$

и $J_{k_{l}}\left(z_{l}, v_{l}\right)$ - жорданова клетка, соответствующая комплексно сопряженным полюсам системы $z_{l} \pm j v_{l}$ кратности $k_{l}$

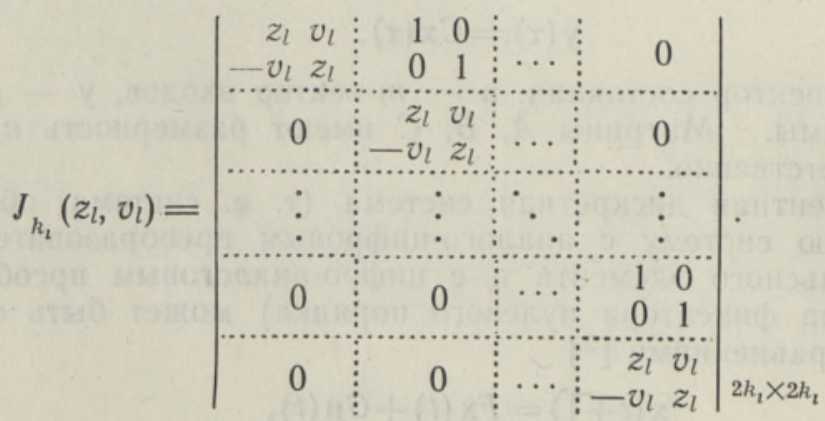

Разделим теперь матрицу $J_{d}$ на 4 блока: два нулевых, $F_{4}$ (содержит малые собственные значения (близкие к нулю по модулю) размерами 
$n_{2} \times n_{2}$ ) и $F_{1}$ (содержит большие собственные значения (близкие к единице по модулю) размерами $\left.n_{1} \times n_{1}\left(n_{1}+n_{2}=n\right)\right)$, как это показано ниже

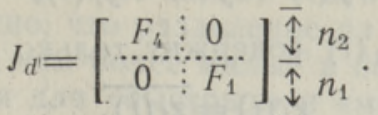

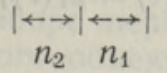

Представим теперь $q(t)$ в виде

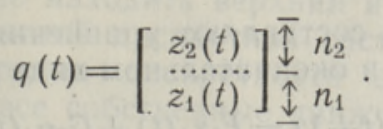

Разделим теперь матрицы $\widetilde{G}$ и $\tilde{H}$ на блоки так, как это показано ниже

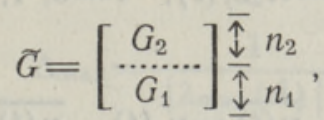

$$
\begin{aligned}
& |\leftrightarrow| \\
& \tilde{H}=\left[\begin{array}{l:l}
H_{2} & H_{1}
\end{array}\right] \underline{\hat{\imath}} p .
\end{aligned}
$$

Следовательно, теперь систему (7)-(8) можно записать в таком виде

$$
\begin{aligned}
& z_{1}(t+1)=F_{1} \mathbf{z}_{1}(t)+G_{1} \mathbf{u}(t), \\
& \mathbf{z}_{2}(t+1)=F_{4} \mathbf{z}_{2}(t)+G_{2} \mathbf{u}(t), \\
& \mathbf{y}(t)=H_{1} \mathbf{z}_{1}(t)+H_{2} \mathbf{z}_{2}(t), \quad t=0,1,2, \ldots .
\end{aligned}
$$

Полученное представление системы (9)-(11) согласно $\left[{ }^{6}\right]$ и является представлением системы (3)-(4) в форме с предварительно разделенными «медленными» и «быстрыми» переменными.

\section{4. Получение уравнений состояния двухскоростного многомерного дискретного объекта}

Для окончательного разделения предварительно разделенных дискретных подсистем (9)-(11) воспользуемся методом, предложенным в $\left[{ }^{7}\right]$, который использует свойство временной разделимости «медленных» и «быстрых» мод дискретной системы. Предположим, что рассматриваются только «медленные» моды (это показывает черта над символами). Тогда система (9)-(11) примет такой вид:

$$
\begin{aligned}
\overline{\mathbf{z}_{1}(t+1)} & =F_{1} \overline{\mathbf{z}_{1}(t)}+G_{1} \overline{\mathbf{u}(t)}, \\
\overline{\mathbf{z}_{2}(t+1)} & =F_{4} \overline{\mathbf{z}_{2}(t)}+G_{2} \overline{\mathbf{u}(t)}, \\
\overline{\mathbf{y}(t)} & =H_{1} \overline{\mathbf{z}_{1}(t)}+H_{2} \overline{\mathbf{z}_{2}(t)} .
\end{aligned}
$$

Вычитая из уравнения (10) уравнение (13) и обозначив

$$
\mathbf{z}_{2}(t)-\overline{\mathbf{z}_{2}(t)}=\mathbf{z}_{f}(t), \quad \mathbf{u}(t)-\overline{\mathbf{u}(t)}=\mathbf{u}_{f}(t),
$$

получим такое уравнение

$$
\mathbf{z}_{f}(t+1)=F_{4} \mathbf{z}_{f}(t)+G_{2} \mathbf{u}_{f}(t) .
$$


Вычитая из уравнения (11) уравнение (14) и обозначив

$$
\mathbf{y}(t)-\overline{\mathbf{y}(t)}=\mathbf{y}_{f}(t),
$$

а также учитывая, что $\mathbf{z}_{1}(t)$ содержит только «медленные» моды, т. е.

$$
\mathbf{z}_{1}(t)=\overline{\mathbf{z}_{1}(t)}
$$

получим

$$
\mathbf{y}_{f}(t)=H_{2} \mathbf{z}_{f}(t)
$$

Уравнения (15) и (16) и составляют уравнения «быстрой» подсистемы, которую можно записать в окончательном виде так:

$$
\begin{aligned}
& \mathbf{z}_{f}(t+1)=F_{4} \mathbf{z}_{f}(t)+G_{2} \mathbf{u}_{f}(t), \\
& \mathbf{y}_{f}(t)=H_{2} \mathbf{z}_{f}(t), \quad t=0,1,2, \ldots .
\end{aligned}
$$

Обозначив

$$
\overline{\mathbf{z}_{1}(t)}=\mathbf{z}_{s}(t), \quad \overline{\mathbf{y}(t)}=\mathbf{y}_{s}(t), \quad \overline{\mathbf{u}(t)}=\mathbf{u}_{s}(t),
$$

можно переписать систему (12)-(14) так:

$$
\begin{aligned}
\mathbf{z}_{s}(t+1) & =F_{1} \mathbf{z}_{s}(t)+G_{1} \mathbf{u}_{s}(t), \\
\mathbf{z}_{2}(t+1) & =F_{4} \overline{\mathbf{z}_{2}(t)}+G_{2} \mathbf{u}_{s}(t), \\
\mathbf{y}_{s}(t) & =H_{1} \mathbf{z}_{s}(t)+H_{2} \overline{\mathbf{z}_{2}(t)} .
\end{aligned}
$$

Считая, что «быстрые» процессы быстро достигают установившегося состояния, а следовательно $\overline{\mathbf{z}_{2}(t)}=$ const, можно переписать систему (17) - (19) так:

$$
\begin{aligned}
\mathbf{z}_{s}(t+1) & =F_{1} \mathbf{z}_{s}(t)+G_{1} \mathbf{u}_{s}(t), \\
\overline{\mathbf{z}_{2}(t)} & =F_{4} \overline{\mathbf{z}_{2}(t)}+G_{2} \mathbf{u}_{s}(t), \\
\mathbf{y}_{s}(t) & =H_{1} \mathbf{z}_{s}(t)+H_{2} \overline{\mathbf{z}_{2}(t)} .
\end{aligned}
$$

Из уравнения (21) получим

$$
\overline{\mathbf{z}_{2}(t)}=\left[I-F_{4}\right]^{-1} G_{2} \mathbf{u}_{s}(t),
$$

если обратная матрица $\left[I-F_{4}\right]^{-1}$ существует. С учетом (23) уравнение (22) можно записать

$$
\mathbf{y}_{s}(t)=H_{1} \mathbf{z}_{s}(t)+H_{2}\left[I-F_{4}\right]^{-1} G_{2} \mathbf{u}_{s}(t) .
$$

Уравнения (20) и (24) и составляют уравнения «медленной» подсистемы, которую окончательно можно записать так:

$$
\begin{aligned}
& \mathbf{z}_{s}(t+1)=F_{1} \mathbf{z}_{s}(t)+G_{1} \mathbf{u}_{s}(t), \\
& \mathbf{y}_{s}(t)=H_{1} \mathbf{z}_{s}(t)+H_{2}\left[I-F_{4}\right]^{-1} G_{2} \mathbf{u}_{s}(t), \quad t=0,1,2, \ldots
\end{aligned}
$$

\section{5. Метод выбора периодов дискретности двухскоростного многомерного дискретного объекта}

Выбор периодов дискретности двухскоростного многомерного дискретного объекта управления зависит от динамик процессов в обеих подсистемах («медленной» и «быстрой»). Чем меньше период дискретности, тем точнее и полнее получаемая информация. При слишком малом периоде дискретности система становится по своим динамическим свойствам квазинепрерывной, но при этом существенно возрастает за- 
грузка ЦВМ. При очень большом периоде дискретности часть информации теряется, поэтому становится невозможным качественно управлять процессом, а сам процесс может стать неуправляемым. С интуитивной точки зрения ясно, что разделение на «медленную» и «быструю» подсистемы тем лучше, чем более малые и более большие периоды дискретности используются для «быстрых» и «медленных» подсистем соответственно. Поэтому при выборе периодов дискретности двухскоростного многомерного дискретного объекта управления необходимо добиваться компромисса между всеми возможностями, а на практике для этого обычно достаточно находить верхний и нижний пределы периода дискретности эквивалентной дискретной системы.

Верхний предел периода дискретности эквивалентной дискретной системы, при котором все собственные движения непрерывной системы точно воспроизводятся эквивалентной дискретной системой, определяется по формуле $\left[{ }^{8}\right]$

$$
\Delta_{\max }=\frac{1}{\left|\lambda_{\max }(A)\right|}
$$

где $\lambda_{\max }(A)$ - максимальное собственное значение матрицы $A$ непрерывной системы (1)-(2). Условие (25) учитывает не только колебательные, но и апериодические составляющие движения системы.

Нижний предел периода дискретности эквивалентной дискретной системы определяется из условия равенства приращения выходного вектора на одном шаге дискретности величине квантования по уровню того же выходного вектора при подаче на вход объекта сигнала управления, равного величине его квантования по уровню.

Пусть задана эквивалентная дискретная система

$$
\begin{aligned}
& \mathbf{x}[(t+1) \Delta]=[F(\Delta)] \mathbf{x}(t \Delta)+[G(\Delta)] u(t \Delta), \\
& \mathbf{y}(t \Delta)=[H(\Delta)] \mathbf{x}(t \Delta), \quad t=0,1,2, \ldots .
\end{aligned}
$$

Из решения системы $(26)-(27)$ при $t=0$ и $x(0)=0$ получим

$$
\mathbf{y}[\Delta]=[H(\Delta)][G(\Delta)] \mathbf{u}[0] \text {. }
$$

Обозначив

$$
[H(\Delta)][G(\Delta)]=[W(\Delta)],
$$

приняв величину вектора управляющего воздействия равной величине его квантования по уровню (которая должна быть известна)

$$
\mathbf{u}[0]=\delta \mathbf{u},
$$

приняв величину $\mathbf{y}[\Delta]$ равной величине квантования выходного вектора $\delta \mathbf{y}$ (которая должна быть известна), получим уравнение для определения нижнего значения периода дискретности $\Delta_{\mathrm{min}}$

$$
\delta \mathbf{y}=\left[W\left(\Delta_{\min }\right)\right] \delta \mathbf{u} .
$$

В полученном уравнении (29) неизвестными являются элементы матрицы $\left[W\left(\Delta_{\min }\right)\right]$, которые зависят от периода дискретности $\Delta_{\min }$. После развертывания уравнения (29) по строкам получим $p$ уравнений и для каждого из полученных уравнений определим свое $\Delta_{j_{\min }}$. Выбирая из полученного ряда $\Delta_{j_{\min }}$ наибольшее, найдем предельное значение $\Delta_{\min }$.

Таким образом, найденное значение $\Delta_{\min }$ и будет являться нижним пределом периода дискретности эквивалентной дискретной системы (26) - (27). Действительно, если взять значение периода дискретности меньше $\Delta_{\mathrm{min}}$, т. е. $\Delta_{0}<\Delta_{\mathrm{min}}$, то приращение координат выходного век- 
тора при воздействии вектора управления, равного по величине одному кванту, не приведет к изменению показаний датчиков на некоторых выходах системы. Следовательно, данное управление будет бесполезным и проявит себя только при нескольких шагах дискретности.

Для нахождения $\Delta_{\min }$ необходимо знать шаги квантования по уровню входного вектора $\delta \mathbf{u ~ и ~ в ы х о д н о г о ~ в е к т о р а ~} \delta$, которые можно определить, исходя из статических характеристик АЦП и ЦАП [ $\left.{ }^{9}\right]$, применяемых при технической реализации данной задачи управления

$$
\delta u_{l}=\frac{u_{l_{\max }}}{2^{\alpha_{l}}-1}, \quad l=1,2, \ldots, m,
$$

где $\alpha_{l}-$ разрядность кода, в котором может быть представлена величина $u_{l}(\tau)$ на выходе ЦАП, $u_{l_{\max }}$ - максимальное значение сигнала $u_{l}(\tau)$

$$
\delta y_{j}=\frac{y_{j_{\max }}}{2^{\beta_{j}}-1}, \quad j=1,2, \ldots, p
$$

где $\beta_{j}-$ разрядность двоичного кода АЦП, $y_{j_{\max }}-$ максимальное значение модуля сигнала $y_{j}(\tau)$ на входе АЦП.

Период дискретности $\Delta$ для эквивалентной дискретной системы (3)-(4) определяется таким неравенством

$$
\Delta_{\min } \leqslant \Delta \leqslant \Delta_{\max }
$$

Здесь $\Delta_{\max }$ определяется по формуле $(25) ; \Delta_{\min }$ определяется из матричного уравнения (29), где величины $\delta \mathbf{u}$ и $\delta$ у определяются формулами $(30)$ и (31) соответственно, а $\left[W\left(\Delta_{\min }\right)\right]$ имеет в соответствии с (28) и (5), (6) такой вид:

$$
\left[W\left(\Delta_{\min }\right)\right]=C\left[\int_{0}^{\Delta_{\min }} \exp (A \eta) d \eta\right] B .
$$

Но согласно $\left[{ }^{5}\right]$ можно записать такое выражение:

$$
\left[\int_{0}^{\Delta_{\min }} \exp (A \eta) d \eta\right]=\left[\exp \left(A \Delta_{\min }\right)-I\right] A^{-1},
$$

при условии, что матрица $A$ - неособая. Для вычисления матричной экспоненты $\exp \left(A \Delta_{\min }\right)$ можно использовать приближение Паде $\left[{ }^{5}\right]$

$$
\exp \left(A \Delta_{\min }\right)=\left(I-\frac{\Delta_{\min }}{2} A\right)^{-1}\left(I+\frac{\Delta_{\min }}{2} A\right) \text {. }
$$

Учитывая (33) и (34), выражение (32) можно записать в таком виде

$$
\left[W\left(\Delta_{\min }\right)\right]=C\left[\left(I-\frac{\Delta_{\min }}{2} A\right)^{-1}\left(I+\frac{\Delta_{\min }}{2} A\right)-I\right] A^{-1} B .
$$

При малых значениях периода дискретности $\Delta_{\min }$ можно воспользоваться такой приближенной формулой для вычисления матричного экспоненциала

$$
\exp \left(A \Delta_{\min }\right) \approx I+A \Delta_{\min }
$$

Теперь выражение (32) можно переписать в таком виде

$$
\left[W\left(\Delta_{\min }\right)\right]=\Delta_{\min } C B \text {. }
$$




$$
C B=\left[\begin{array}{cccc}
v_{11} & v_{12} & \ldots & v_{1 m} \\
v_{j 1} & v_{j 2} & \ldots & v_{j m} \\
v_{p 1} & v_{p 2} & \ldots & v_{p m}
\end{array}\right]
$$

мы можем определить значение $\Delta_{j_{\min }}$ после развертывания уравнения (29) по $j$-ой строке таким образом

$$
\Delta_{j_{\text {min }}}=\frac{\delta y_{j}}{v_{j 1} \delta u_{1}+v_{j 2} \delta u_{2}+\ldots+v_{j m} \delta u_{m}} .
$$

Для более точного определения $\Delta_{j_{\min }}$ можно воспользоваться формулами (29) и (35). Можно предложить выбирать периоды дискретности многомерного дискретного объекта управления такими

$$
\Delta_{f} \approx \Delta_{\min }, \quad \Delta_{s} \approx \Delta_{\max } .
$$

\section{6. Заключение}

Разработан метод получения описания в пространстве состояний двухскоростного многомерного дискретного объекта управления исходя из представления эквивалентной дискретной системы в канонической форме Жордана. Предложен также метод выбора периодов дискретности двухскоростного многомерного дискретного объекта, который позволяет находить эти периоды дискретности, исходя из известных уравнений в переменных состояния двухскоростного многомерного дискретного объекта и статических характеристик преобразования АЦП и ЦАП, применяемых при технической реализации данной задачи управления.

\section{ЛИТЕРАТУРА}

1. Kando, H., Iwazumi, T. // Int. J. Control., 1986, 44, № 6, 1555-1578.

2. Chandrasekharan, P. C., Sambandan, A. // Control and Cybernetics., 1984, 13, № 1-2. $27-38$.

3. Квакернаак X., Сиван Р. Линейные оптимальные системы управления. М., Мир, 1977.

4. Изерман Р. Цифровые системы управления. М., Мир, 1984.

5. Воеводин В. В., Кузнецов Ю. А. Матрицы и вычисления. М., Наука, 1984.

6. Oloomi, H., Sawan, M. E. // IEEE Trans. Automatic Control., 1987, 32, № 3, $246-248$.

7. Litkouhi, B., Khalil, H. // Proc. Amer. Contr. Conference. California, 1984, 3, $1385-1390$.

8. Аверина А. Д., Герасимов А. Н., Забродин C. П. Дискретные нелинейные системы. М., Машиностроение, 1982.

9. Бахтиаров Г. Д., Малинин В. В., Школин В. П. Аналого-цифровые преобразователи. М., Советское радио, 1980. 


\section{ASTROV}

\section{KAHEKIIRUSELISE MITMEMOOTMELISE DISKREETSE OBJEKTI OLEKUMUDEL}

Dünaamilise süsteemi olekumudel on Jordani kanoonilist kuju kasutades dekomponeeritud aeglaseks ja kiireks alamsüsteemiks. On määratud vastavate alamsüsteemide diskreetimise perioodide alumised ja ülemised piirid.

\section{ASTROV}

\section{STATE EQUATIONS FOR TWO-RATE MULTIVARIABLE DISCRETE-TIME SYSTEMS}

The behaviour of the systems having a time-separation property is approximated by two lower-order subsystems: a slow subsystem with large eigenvalues and a fast subsystem with small eigenvalues. The method proposed in this paper permits finding state equations for two-rate multivariable systems if equivalent discrete-time system (3)-(4) is presented in canonical Jordan's form (7)-(8). State equations for fast and slow subsystems are given by (15), (16) and (20), (24). respectively. High and low limits of sampling periods for slow and fast discrete-time subsystems have been obtained. 